CARNETS OE Carnets de géographes

GÉOGRAPHES

\title{
(Ap)prendre la géographie par les sentiments
}

L'apprentissage d'une géographie sensible à partir des émotions

littéraires

Fabienne Cavaille

\section{(2) OpenEdition}

Journals

Édition électronique

URL : http://journals.openedition.org/cdg/565

DOI : $10.4000 /$ cdg. 565

ISSN : 2107-7266

Éditeur

UMR 245 - CESSMA

Référence électronique

Fabienne Cavaille, «(Ap)prendre la géographie par les sentiments», Carnets de géographes [En ligne], 9 | 2016, mis en ligne le 30 novembre 2016, consulté le 19 avril 2019. URL : http://

journals.openedition.org/cdg/565; DOI : 10.4000/cdg.565

La revue Carnets de géographes est mise à disposition selon les termes de la Licence Creative Commons Attribution - Pas d'Utilisation Commerciale - Pas de Modification 4.0 International. 
(AP)PRENDRE LA GEOGRAPHIE PAR LES SENTIMENTS :

L'apprentissage d'une géographie sensible à partir des émotions littéraires

FABIENNE CAVAILLE

Maître de conférences, Département de Géographie, LISST - Dynamiques Rurales

fabienne.cavaille@univ-tlse2.fr

\title{
Résumé
}

Ce texte envisage la manière de familiariser les enfants avec une géographie des émotions à partir de la littérature de jeunesse. Les œuvres de littérature de jeunesse de fiction constituent en effet des supports et des opportunités privilégiés pour une découverte des émotions géographiques. L'objectif visé est de proposer des activités concrètes à mener sur le terrain auprès d'enfants du niveau de l'école primaire - à l'âge où leur "géographicité " se structure. L'apport essentiel de ce texte consiste ainsi en la présentation d'une démarche décomposant les étapes de travail sur un corpus d'œuvres de fiction et sur un album plus particulièrement, concernant la gamme des émotions ayant trait à l'expérience de la migration et de l'exil (le malheur, la surprise, la sécurité, le bonheur). En préalable, est posé le cadre d'intelligibilité des émotions sur lequel se fonde la démarche appliquée. Selon celuici, il s'avère que ce sont les sentiments, en tant que soubassement des émotions, qu'il est intéressant de mettre au jour.

Mots-clefs: émotions, sentiments, apprentissage de la géographie, littérature de jeunesse, exil

\begin{abstract}
This study considers how to introduce an emotional geography to children and pupils. Fictional children's literature is used as object and support preferred to propose such learning (in primary school level, at the time sense of geographicity is in full development,). Firstly, this text submits a theoretical framework. Under this, emotions are based on feelings (and sentiments) that appear as the most interesting to reveal and to study. Secondly, a practical process to investigate geographic emotions with children's literature is proposed. The main purpose of this paper is to offer a set of concretes activities for a field work within the school curriculum or in extracurricular activities. A fiction's corpus (in particular a picture book) about migration and exile's experiences is taken as an example to literary and geographical works. A range of exile's emotions (sadness, surprise, peace, security, happiness) is identified.
\end{abstract}

Keywords: emotions, feelings, leaning geography, children's literature, exile 


\section{Introduction}

"Tant que ces travaux sur la sensibilité nous feront défaut, il n'y aura pas de géographie possible ${ }^{1}{ }^{1}$

Le champ d'une géographie sensible, notamment d'une géographie des émotions s'est progressivement imposé dans le domaine de la recherche. Il s'est nourri pour cela des avancées théoriques et méthodologiques de plusieurs autres disciplines. Ici, c'est au travers des œuvres littéraires et des expériences de lecture qu'est appréhendée cette géographie des affects. L'objectif est plus précisément de promouvoir ce champ de la géographie auprès des enfants, dans des situations d'apprentissage. II semble pertinent de proposer les acquis de la recherche jusqu'aux enfants, au moment où leur "sentiment géographique ", leur " géographicité » se structure. II s'agit ainsi d'envisager comment la littérature de jeunesse peut être le support et l'opportunité de découverte et d'apprentissages d'une géographie qui s'attache aux vécus affectifs de l'espace. Cette contribution correspond à la petite fabrique d'une recherche appliquée, en vue de propositions concrètes d'activités auprès d'enfants, en classe ou dans des ateliers de lecture. L'apport essentiel de ce texte consiste en la présentation d'une démarche décomposant les étapes de travail sur un corpus d'œuvres de fiction et sur une œuvre choisie plus particulièrement, permettant de s'initier directement à une géographie sensible. Les supports sélectionnés concernent la gamme des émotions géographiques liées à l'expérience de l'exil. Avant cela, il est important de poser des éléments de cadrage épistémologique des émotions, en s'attachant à la spécificité du travail sur la littérature.

\section{Emotions géographiques, littérature et apprentissages}

Plusieurs constats, relativement bien connus ${ }^{2}$, justifient cette recherche. Pour une part, la géographie a pris en compte les émotions au travers d'une variété d'objets, de questions, de populations étudiées, voire des méthodes mobilisées et même au travers des expériences personnelles du chercheur. Chaque fois que des géographes se sont attachés à démontrer que la relation à l'espace a une importance pour les individus et les groupes, parce qu'elle conditionne leur vie quotidienne, qu'elle est le support de leurs projets, qu'elle renferme leurs histoires personnelles et collectives, qu'elle explique leur bien-être ou leur mal être, ils ont été amenés à mettre au jour, d'une manière ou d'une autre, des émotions, des affects, des sentiments géographiques. Certains intérêts de recherche, tels les trajectoires de vie d'habitants ou encore les mobilisations collectives et les conflits (par exemple autour de l'expropriation, Cavaillé, 1999) les y ont de fait beaucoup plus directement confrontés et les affects sont alors devenus des objets de recherche en soi (Feildel, 2011).

II serait probablement assez facile de démontrer que les émotions ont toujours été présentes dans la géographie française. On retrouverait par exemple peut-être assez systématiquement le motif du " merveilleux » éprouvé face à l'œuvre de la nature et des

\footnotetext{
${ }^{1}$ II s'agit du détournement du texte de Lucien Febvre qui, dès 1941, encourageait vivement ses collègues historiens « à faire une place dans leurs recherches et leurs explications aux sentiments, passions ou émotions collectives et singulières des hommes: "Tant que ces travaux nous feront défaut, il n'y aura pas d'histoire possible" » (Mandrou, 1959 : 581).

${ }^{2}$ Voir l'appel à contribution pour ce numéro.
} 
hommes dans les textes "classiques " avec une volonté de faire naître un émoi chez le lecteur (plusieurs passages du célèbre Tableau de la géographie de la France de Paul Vidal de la Blache en témoignent). D'autres courants de la discipline valorisant les " espaces vécus " et/ou une "géographie humaniste " ont, dès les années 1970, fait explicitement référence aux sentiments et aux affects. Ainsi A. Frémont a-t-il défendu au fil de ses travaux une analyse des lieux combinant une "trame objective » et un intérêt pour les sensibilités: " La sensibilité du corps et de l'esprit, les cinq sens, l'affect, la mémoire, le sens esthétique, l'émotion, la révolte ou le rêve, sans que cette énumération soit exhaustive, y comptent au moins autant que l'exercice de la raison " (Frémont, 2010 : 165). II faudrait s'arrêter sur le fait que pour ce chercheur et surtout pour d'autres (notamment Levy, 1997 ; Bédard, 1987 ; Mattey, 2008), la littérature a joué un rôle essentiel dans la découverte et l'affirmation de ce champ de la géographie.

Toutefois, sans y insister ici, il pourrait également être démontré que de nombreux géographes français restent réticents à l'égard des émotions, qu'ils les évitent, en font un objet non représentatif du cœur de la discipline et relèguent donc à la marginalité les travaux qui s'y consacrent. Le même A. Frémont pense que :

"ne voir dans les lieux que des pulsions, des émotions, des représentations, des perceptions, c'est finalement ne retenir que des images de soi ou des proches, sans intérêt pour la matière et la manière qui les ont produites, c'est aller au-devant des bavardages, des anarchies, des communautarismes et des fantasmes » (lb.).

On peut observer, sans l'expliciter non plus, que cette défiance (combinée à une certaine méconnaissance) se reproduit pour une part dans l'enseignement de la discipline, en particulier dans le premier et le second degré. L'introduction dans les programmes de géographie (école et collège notamment) du concept de "l'habiter " ou plus récemment dans les programmes pour le français de thèmes comme "les territoires de l'imaginaire » ont certes constitué une étape importante. Néanmoins, le champ d'une géographie sensible gagnerait encore à être mieux défini en tant qu'objet d'enseignement et pour ce faire à être mieux connu en tant qu'objet scientifique. Les "transpositions didactiques " mériteraient d'être consolidées de manière à ce que les expériences sensibles et existentielles des acteurs et des sujets concernant l'espace soient effectivement prises comme objets d'apprentissage auprès des élèves et des enfants-habitant (ce à quoi contribue notamment J-F. Thémines par exemple avec Le Guern, 2011).

Cette démarche s'avère d'autant plus opportune et légitime que des didacticiens et diverses disciplines ont explicitement affirmé l'intérêt de travailler en situation d'apprentissage sur et avec les émotions et les affects. L'intérêt pour les émotions relève de différentes préoccupations: utiliser les émotions pour intéresser et impliquer les élèves dans les activités, amener les enfants à mieux connaître et à mieux maîtriser leurs émotions c'est-àdire à les développer, du moins certaines, pour être créatifs, pour développer un sens critique et pour participer à leur propre édification en tant que sujet, mais aussi à les contrôler quand elles deviennent trop envahissantes (générant alors des violences) (par exemple Puozzo Capron, Piccardo, 2013). Cet intérêt éducatif et pédagogique pour les émotions s'est de fait, et de manière ancienne, porté sur la littérature, sur les activités de lecture et d'écriture. Les programmes scolaires et les documents officiels de 2002 ont manifestement été assez déterminants : à travers la mise en avant de la littérature de jeunesse, une grande place est donnée aux affects, ceux des personnages, voire de l'auteur, et ceux du lecteur, amené à mobiliser et à exprimer sa sensibilité. Les activités de maîtrise de 
la langue comportent elles aussi un travail utilisant la subjectivité du scripteur. Diverses disciplines sont concernées. C'est le cas en particulier d'historiens pour qui les sensibilités sont un objet ancien et dont un certain nombre s'intéresse (Jaubert et al. 2013) et est favorable à l'utilisation des fictions de jeunesse pour l'apprentissage de leur matière, notamment parce qu'elles permettent une dynamique chargée "d'affects et d'empathie " (Cariou, 2012 : 177).

Du point de vue de la géographie, en tant qu'objet de recherche et d'enseignement, les émotions peuvent être différenciées selon trois niveaux. Peuvent d'abord être identifiées et étudiées les émotions géographiques en tant que telles c'est-à-dire les réactions éprouvées en lien direct avec l'espace, un lieu, un paysage, etc. du fait de ses caractéristiques, et des relations avec le sujet qui les éprouve, par exemple la peur ou l'anxiété vis-à-vis d'un espace inconnu, ou mal aimé, le bien-être dans un espace intime ou personnalisé, l'excitation lors de la découverte d'un nouvel espace, etc. Pourraient ensuite être distinguées des émotions géographiques médiatisées c'est-à-dire les réactions qui ont pour objet un motif tiers, qui n'ont pas directement trait à un lieu particulier, par exemple la colère ressentie à l'occasion d'un conflit environnemental très éloigné (la réaction est liée à la cause environnementale et non à l'environnement affecté), la nostalgie du pays d'origine éprouvée à la lecture d'un courrier (la réaction porte sur un souvenir), etc. Peuvent enfin être dissociées les émotions géographiques comme mode d'intelligibilité. II s'agit d'émotions d'un autre ordre, consubstantiel aux deux premiers qui sont celles du sujet (chercheur, élève, enseignant) qui lui permettent la compréhension et la connaissance des faits spatiaux. Ce sont les processus et ressorts intellectuels et cognitifs d'ordre affectif qui sont mobilisés et qui reposent notamment sur l'empathie, la réflexivité.

En situation d'apprentissage en géographie, différentes modalités peuvent a priori être privilégiées pour faire découvrir et faire travailler de telles émotions aux enfants et aux élèves : l'enquête in situ, sous différentes formes, l'intervention et le témoignage d'acteurs, la recherche documentaire, ou bien encore la littérature de jeunesse. Celle-ci est apparue comme une richesse infinie lors de travaux exploratoires - consistant à la fois en des interventions auprès d'étudiants de l'ESPE (Ecole supérieure du professorat et de l'éducation), des analyses de supports d'enseignements, des études de corpus de littérature de jeunesse et des échanges avec des enseignants du primaire. L'exploration de corpus a révélé qu'une " géographicité ", c'est-à-dire le sentiment d'une relation complexe entre les individus et l'espace, est très prégnante dans la littérature de jeunesse et transparaît très souvent à partir d'expériences émotionnelles marquées.

Ainsi, le rapport à l'espace habité (à travers la recherche, la construction, l'absence ou la perte d'un chez soi, le sentiment d'un bien-être, le partage forcé d'un espace domestique, etc.) (par exemple Frouillou, 2011) mais aussi la vie dans la nature et avec les éléments naturels (leurs risques et les connaissances magiques pour les affronter), les vicissitudes du voyage (l'errance et les rencontres), ou encore le vécu de l'éloignement et de la distance (de la migration et de l'exil), les rites et épreuves de passage inscrits dans les lieux, etc., constituent autant de révélations des émotions géographiques. Peuvent ainsi être travaillées de très nombreuses œuvres de fiction destinées aux enfants, y compris pour les plus jeunes, qui s'offrent avec une extraordinaire diversité de supports - dont les albums, pour lesquels on peut se reporter au très beau travail de Ch. Meunier pour s'en rendre compte). En outre, les contenus effectivement enseignés dans les classes, les compétences transversales visées, la polyvalence et l'interdisciplinarité des enseignants dans le premier degré, permettent 
effectivement d'envisager un apprentissage assez tôt dans la scolarité, à partir de la lecture d'œuvres littéraires, de cette géographicité (Meunier, 2014: 398 et suiv.) et des vécus sensibles qui y sont liés.

La démarche qui est proposée ici consiste donc à utiliser les œuvres de littérature de jeunesse dans lesquelles sont prégnantes des émotions géographiques. Des activités sur les œuvres sont envisagées en situation scolaire, à partir de séquences d'enseignement mais elles peuvent également être proposées dans le cadre péri scolaire (groupes de lecture en centre de loisirs rattachés aux écoles) ou non scolaire (initiatives des bibliothèques municipales). Dans la démarche qui sera présentée, l'œuvre de littérature de jeunesse est centrale et le travail de lecture est premier et structurant - le document y est respecté en tant qu'œuvre. La familiarisation avec une géographie des émotions s'effectue à partir d'un travail de lecture sélectif, orienté et informé par la géographie et à partir d'activités de prolongement de la lecture et de l'œuvre qui relèvent de la géographie. Compte tenu de l'importance accordée dans le cadre scolaire aux apprentissages fondamentaux tels que la lecture, la maîtrise de la langue, parfois au détriment de certaines autres matières, combiner des activités en littérature, français et géographie constitue probablement pour cette dernière une opportunité de se voir requalifiée.

Avant d'envisager la démarche de travail, il nous faut d'abord clarifier a minima d'un point de vue théorique ce que sont les émotions, et notamment dans des expériences de lecture.

\section{Un cadre épistémo-didactique}

La clarification esquissée dans ce qui suit consiste en un rapide bilan épistémologique intégrant les apports de plusieurs disciplines dans l'objectif d'élaborer in fine une grille d'intelligibilité des émotions directement remobilisable pour des expériences de lecture.

Le terme émotions pourrait être utilisé comme le terme générique, mobilisé à la fois par la plupart des sciences humaines et sociales et biologiques et par la plupart des langues européennes et les Anglo-saxons (Nagy, 2013). II recouvrirait alors un large domaine d'études qui s'intéressent aux expériences affectives des individus, à la fois intimes et collectives (des notions voisines pourraient y être assimilées : affects, expériences ou vécus sensibles notamment). Mais le concept d'émotions est ici défini et utilisé selon une acception plus restreinte. II est conçu comme un niveau de vécu intégré dans une armature d'expériences qui comprend a priori trois instances principales : les émotions elles-mêmes, les sentiments et les évaluations éthiques - auxquelles il faut associer les sensations et les passions collectives.

Les émotions sont envisagées, de manière assez commune, comme des états affectifs intenses, caractérisés par une réaction plus ou moins brutale et une durée relativement brève, conditionnée par un objet identifiable (une situation, une personne, une pensée, un comportement, etc.). Ce sont des réactions a priori non intentionnelles, non complètement maîtrisées. Elles correspondent à une décharge, à un élan d'énergie, certains auteurs considérant d'ailleurs qu'elles sont essentiellement tournées vers l'action (la peur pour la fuite), du moins la motivation (Livet, 2004 : 56). C'est en cela qu'elles constituent une dynamique d'engagement des enfants dans certaines activités, en particulier qui génèrent des capacités réflexives. 
Les émotions sont à la fois individuelles et collectives. Elles sont nécessairement éprouvées de manière individuelle, l'individu est affecté personnellement, intimement mais elles peuvent être partagées et sont pour une part transmises socialement, culturellement. Elles correspondent à des normes, des codes sociaux, y compris pour une part dans leurs manifestations physiologiques. Elles sont la manifestation (parfois obligatoire, selon les ethnologues) de l'appartenance à un groupe, à une communauté. II est alors possible d'identifier des "régimes d'émotion ", c'est-à-dire des émotions dominantes propres à une société, un groupe, une époque, etc. Plusieurs auteurs ont insisté sur cette composante sociale et explicité le concept de communauté émotionnelle qui y est associé (Boquet, 2013). Or de fait, ce processus collectif et social est inhérent à de nombreuses situations d'apprentissage et à tout projet pédagogique et didactique. II est alors important de prévoir et de structurer les moments où s'élaborent et se cristallisent ces émotions partagées.

Selon ce cadre de compréhension, les émotions ont pour soubassement les sentiments. Ce sont eux qui motivent et structurent fondamentalement et intimement les émotions. Ils sont d'une certaine manière le soubassement caché des émotions qui, elles, en sont la face visible et explicite. Les sentiments sont le ressenti intime et profond des individus, correspondant aux attitudes et aux pensées durables, stables, des personnes (voir les " émotions dispositionnelles" de Livet, 2004 : 49). Les sentiments relèvent à la fois de l'intériorisation profonde de normes sociales et de positionnements personnels; ils pourraient être assimilés au "for intérieur" de l'individu - se rapprochant ainsi probablement du sens commun. Cette conception associe les sentiments à une "valorisation du moi » et à un mouvement de "retour réflexif sur soi » (Petit, 2014). En étant intimement ancrés dans l'individu, les sentiments peuvent être malgré tout assez indécis puisqu'au carrefour de plusieurs influences sociales, culturelles, entre volonté idéale et possibilité d'action. II faut donc, en situation d'apprentissage, garantir d'une manière ou d'une autre ces possibilités d'expression personnelle des sentiments.

Enfin, les émotions et à travers elles, les sentiments, sont motivées et conditionnées par des normes et des valeurs supérieures, des jugements moraux et des principes civiques, que l'on appellera ici des évaluations éthiques. Selon de nombreux auteurs, dès que l'on tente de comprendre ou même de décrire une émotion, il y a un jugement, une évaluation quant aux comportements adéquats, aux pensées acceptables. C'est une évaluation pour soi, pour soi avec les autres, en société, et les débats et délibérations portent sur des définitions du juste et de l'injuste, du bon, du beau, du bien et du mal. Si les émotions sont si déterminantes, c'est donc parce qu'elles reposent et sont motivées par des valeurs et des principes supérieurs.

"L'émotion aurait ainsi pour rôle de focaliser l'attention sur les évènements importants de notre vie. [...] Un raisonnement sans émotion, c'est un raisonnement dans un paysage totalement plat qui ne permet pas de sélectionner la bonne solution parmi les solutions possibles. " (Syssau, 2006 : 52, 56).

Les émotions peuvent réellement apparaître comme des modes de comportements et d'action, de compréhension et d'apprentissage. Elles sont ici conçues comme l'une des trois instances imbriquée aux deux autres que sont les sentiments et les évaluations éthiques. Dans ce cadre d'intelligibilité, il semble que les sentiments soient au cœur de la dynamique, étant la base structurante des émotions et accessibles aux individus par leur réflexivité.

II convient de compléter cette armature de base par deux autres composantes: les sensations d'une part, les passions collectives d'autre part. Les premières sont les 
expériences sensorielles, elles accompagnent les émotions avec des fonctions différentes (selon les cas et les situations, en les provoquant, les accentuant ou les atténuant). Les passions collectives sont de méta réactions sociales, regroupant les communautés autour d'un évènement et qui peuvent ponctuellement orienter les évaluations éthiques. Par conséquent, pour étudier les émotions, c'est finalement un emboîtement assez complexe qu'il faudrait prendre en compte impliquant sensations, émotions, sentiments, évaluations éthiques, passions collectives (Figure 1).

Figure 1 : L'étude des émotions

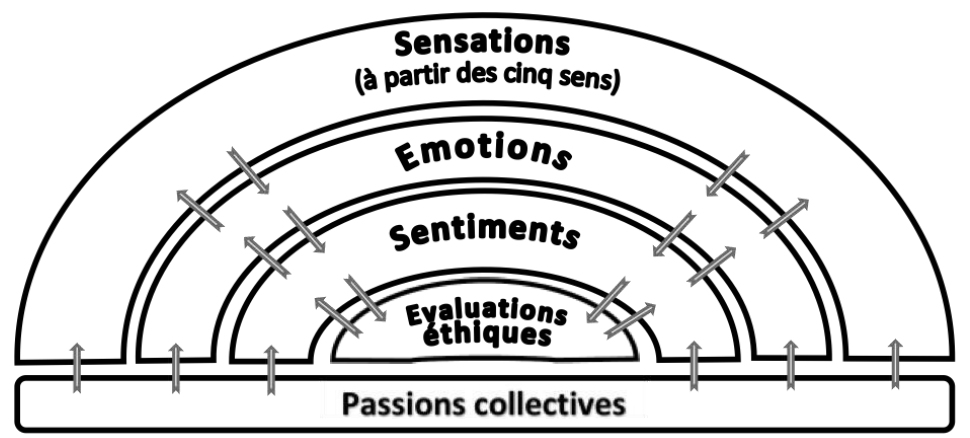

Réalisation : F. Cavaillé, 2016.

Ces repères épistémologiques constituent le cadrage général à travers lequel vont être considérées les émotions littéraires et leurs spécificités.

\section{Les émotions dans les expériences de lecture}

Il s'agit de proposer, ici aussi, une approche globale et analytique des émotions en littérature permettant par la suite d'envisager concrètement le travail sur les œuvres.

Le premier ordre d'émotions littéraires est d'abord bien sûr celui des émotions issues de l'œuvre, des évènements du récit, et celles qui sont directement vécues par le(s) personnage(s). Ce sont les "émotions manifestées", exprimées et représentées dans l'œuvre; elles sont, plus ou moins directement, effectivement, partagées par le lecteur (Jouve, 2010/2014 : 125-126). Elles sont fréquemment issues ou transmises par des " effets de style » et dans la littérature de jeunesse, elles sont très souvent relayées voire suggérées par des illustrations.

Le second ordre d'émotions, qui est de fait consubstantiel au premier, est celui des émotions du lecteur en train de lire, c'est-à-dire des émotions qui accompagnent la lecture. Ce sont les "émotions ressenties" ou "générées" (id.). Elles s'expliquent par le fait que l'acte de lecture en lui-même, le plus simple, génère et mobilise des émotions car il se met en place :

« un processus interactionnel à travers lequel un lecteur empirique réagit affectivement aux sollicitations d'une œuvre en puisant dans sa mémoire intime, sa personnalité profonde, sa culture singulière, son imaginaire propre, c'est-à-dire [une] activité créatrice du sujet lecteur [...]. Prennent alors une grande importance [...] de simples détails qui, en interpellant ponctuellement le lecteur dans ses souvenirs, ses goûts ou ses désirs, génèrent une émotion de lecture souvent intense " (Langlade, $2014: 48,49$ ).

On peut préciser que si les émotions peuvent être très fortes, elles sont souvent (le plus souvent ?) relativement superficielles c'est-à-dire qu'elles ne produisent pas de grands 
bouleversements psychologiques, physiologiques. Mais il y a bien une affectation qui engage le lecteur et qui interpelle ses sentiments. Ainsi « l'émotion générée (en conformité ou non avec l'intention de l'auteur) a [...] sa valeur propre : elle nous éclaire sur la relation affective particulière que nous (lecteurs) entretenons avec une idée, une pensée ou un objet du monde » (Jouve, 2010/2014 : 126-127). L'émotion est alors de fait plus ou moins explicite et plus ou moins consciente.

Un troisième ordre d'émotions correspond aux émotions lorsqu'elles sont analysées, " travaillées " par le lecteur et qu'elles « travaillent » le lecteur à tel point que son existence en est transformée (Figure 2). Cela s'explique par le fait que le lecteur est invité :

"[...] à participer à des expériences de pensée d'une certaine sorte, dans lesquelles des personnages inventés se trouvent placés dans des situations hypothétiques qui exigent d'eux des décisions qui sont la plupart du temps à la fois difficiles et lourdes de conséquences, et à nous demander avec eux: quelle serait la bonne façon d'agir dans une situation de cette sorte? 》 (Bouveresse, $2006: 140)$.

Figure 2 : Les émotions littéraires

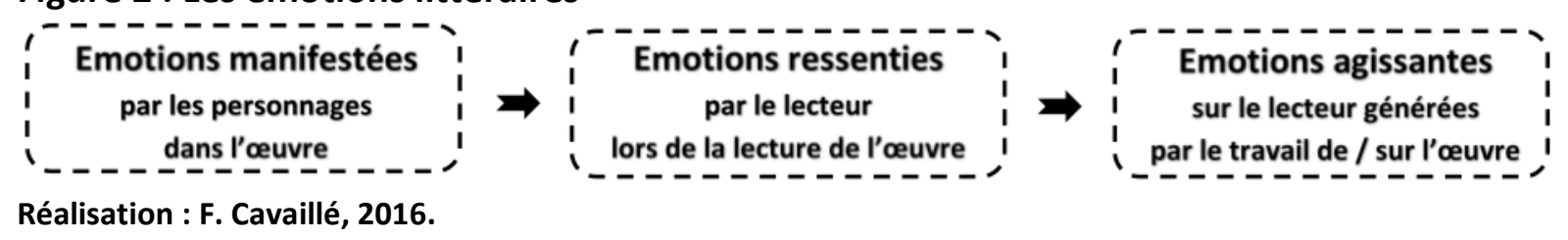

Ainsi, l'expérience de lecture consiste en des épreuves renouvelées d'interprétations et de délibérations sur sa propre existence, son mode et ses principes de vie. La lecture est alors de fait conçue comme un "travail " c'est-à-dire un investissement et un engagement, qui s'effectue à partir des expériences fictives des œuvres mais également comme posture devenue très soucieuse et très attentive aux " styles " de vie, qui les jauge, les hiérarchise (Darsel, 2012/2014; Macé, 2014). La lecture devient de ce fait « une technique sociale du sentiment " (Nonnon, 2008) qui extériorise et objective les intérêts et les mobiles sociaux des individus.

On retrouve ici la dynamique éthique des émotions. Dans nombre de travaux, y compris ceux qui s'intéressent à la littérature de jeunesse, la portée éthique de la littérature, à travers la sensibilité qu'elle véhicule, est manifeste. Les œuvres et activités de lecture constituent des outils de médiation irremplaçables, entre l'individu et ses habitudes, entre un collectif de lecteurs et ses valeurs (Chabanne et al., $2004: 81$ ). L'émotion présente dans la littérature a "une fonction éthique », elle agit "comme déclencheur de prise de conscience de problèmes inaperçus ", comme " un déclencheur de délibération éthique et politique [car elle est] un outil, un médium qui rend capable de percevoir le problème moral, qui prépare au dilemme resté sans elle inaperçu » (Leichter-Flack, 2012/2014). Considérés ainsi, la littérature et le travail sur la littérature ne feraient alors d'ailleurs rien moins que redéfinir la nature de l'éthique et de la morale.

Ces deux armatures serviront de base au travail effectif en situation d'apprentissage concernant les émotions géographiques présentes dans la littérature de jeunesse. 


\section{Le travail sur les émotions géographiques en situation d'apprentissage}

Pour envisager une mise en œuvre concrète, l'ensemble de la démarche est décomposée (Figure 3). Il est pertinent, pour l'élaborer, de s'appuyer sur les acquis de l'enseignement de la littérature de jeunesse (notamment Tauveron, 1999, 2002a, 2002b) mais également sur ceux des autres disciplines, la philosophie (Chirouter, 2013) et l'histoire (notamment Cariou, 2012).

Figure 3 : La démarche générale de travail sur des œuvres de littérature de jeunesse

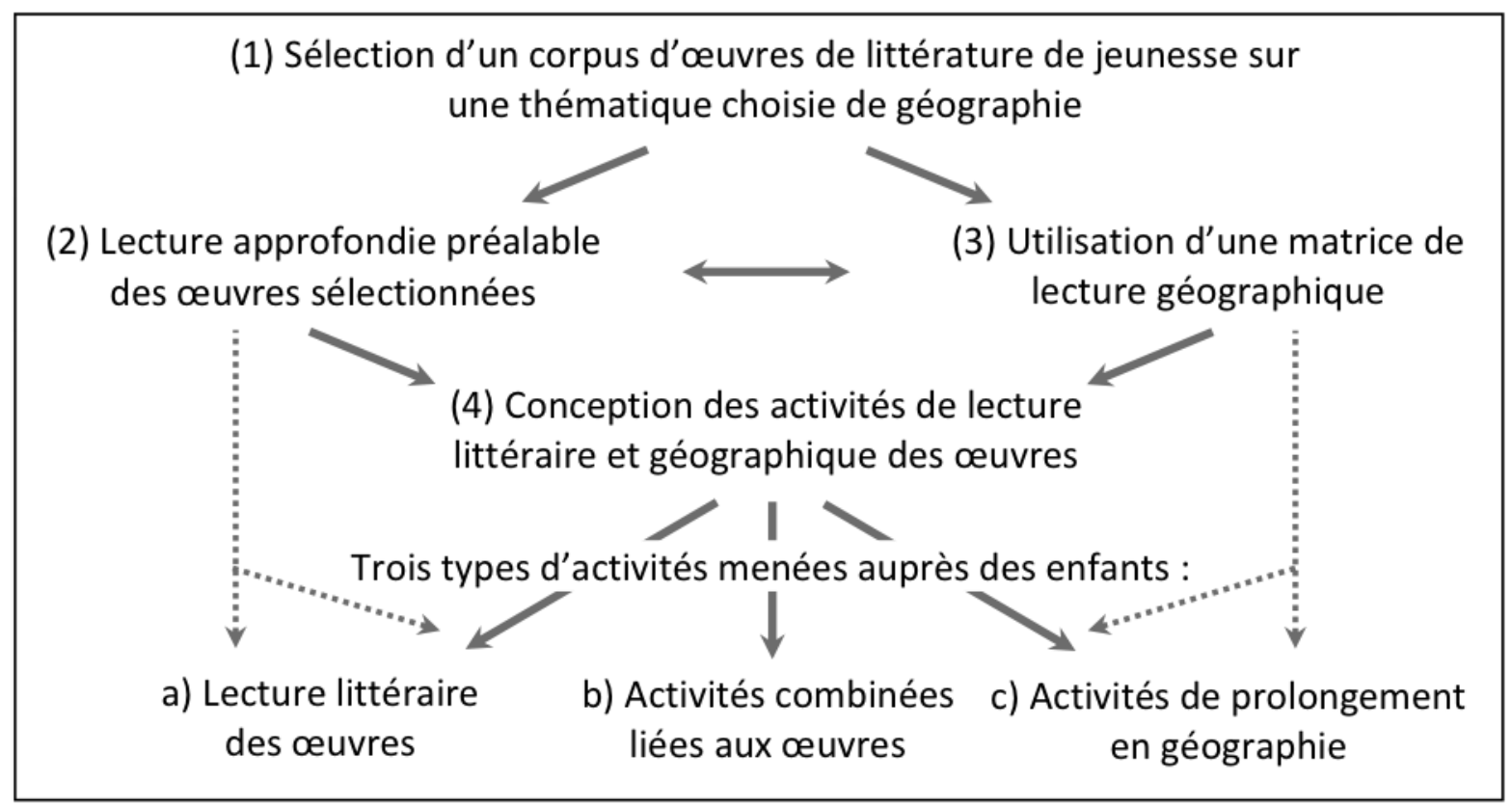

Réalisation : F. Cavaillé, 2016.

La première étape consiste à sélectionner un corpus d'œuvres en relation avec le choix d'une thématique de géographie sensible. Etant donné le foisonnement de la littérature de jeunesse, il est assez aisé de trouver des œuvres sur un objet choisi, et de découvrir des supports de différente nature : album, roman, bande dessinée, théâtre, poésie, etc. Pour faciliter la sélection, il est possible d'utiliser les nombreuses bibliographies existantes (sites et bulletins de bibliothèques, sites pédagogiques d'enseignants, du Ministère de l'Education nationale, etc.) et il est aussi très pertinent de fréquenter directement les ouvrages et les enfants lecteurs. Mieux vaut en effet que les œuvres sélectionnées inspirent de l'intérêt et du plaisir le plus largement possible. Sont retenus les récits qui peuvent faire l'objet d'une lecture littéraire c'est-à-dire ceux qui, parce qu'ils sont " réticents " (Tauveron, $1999:$ 19), interpellent les lecteurs et les invitent à formuler des hypothèses interprétatives. La constitution d'un corpus permet une lecture en réseau c'est-à-dire une lecture qui met en écho plusieurs œuvres de mêmes auteurs ou sur le même thème, amenant ainsi les rapprochements, les éclairages croisés, donnant la possibilité d'aborder de manière relativement complexe et complète une question, favorisant la mémorisation, et participant finalement d'une culture littéraire, d'une culture commune. La lecture en réseau est pratiquée dans les classes et proposée par certaines bibliothèques - et recherchée par certains jeunes lecteurs. Combien il serait désormais pertinent que les géographes contribuent à la constitution de corpus thématiques (comme cela est fait par les historiens) ! 
L'exemple qui est présenté ici est un corpus concernant l'expérience de l'exil. Cette thématique a été retenue car elle révèle $a$ priori des géographies sensibles : les liens intimes et collectifs aux lieux, les expériences d'attachement et d'arrachement aux territoires du quotidien et ce, de manière plurielle, à différentes échelles, selon différentes temporalités (Figure 4). Elle constitue également une problématique vive, récurrente dans l'actualité. Ce corpus est constitué de cinq œuvres de nature fort différente : deux albums, deux romans et une pièce de théâtre. Sans doute peut-on qualifier ces cinq fictions de "réalistes " puisqu'on y retrouve aisément des processus soumis au "réel » et que des repères pris dans "la réalité " sont nécessaires à la compréhension de la fiction (Bruguière, Triquet, 2012). Globalement le corpus peut être proposé à des enfants en fin de cours élémentaire ou durant le cours moyen; les albums peuvent être travaillés dès le cours préparatoire, les romans et la pièce de théâtre plutôt à partir du cours moyen (enfants de 8 à 12 ans). Les principaux personnages en sont des enfants (des filles pour l'essentiel) qui doivent quitter leur milieu d'origine. Selon les documents, c'est l'ensemble ou seulement certaines étapes de l'expérience de l'exil qui sont mises au jour.

Pour rendre plus explicite la démarche, celle-ci va s'attacher à l'une des œuvres du corpus, La cabane d'Isabel (album des auteur et illustrateur américains Sarah Stewart et David Small, publié aux Editions Syros en 2012) - dans le cadre d'une mise en réseau, cet album peut constituer la première œuvre à travailler. Cet album présente l'émigration d'Isabel et de sa famille, du Mexique au Nord des Etats-Unis à la fin des années 1950. La toute jeune fille n'aura de cesse, dès son départ, de vouloir apprendre sa nouvelle langue puis, surtout, de se construire un espace à elle, une cabane de cartons qui lui procurera tranquillité et sécurité. Cet album pourra être directement mis en parallèle ensuite avec un autre album, des mêmes auteur et illustrateur, Le jardin secret de Lydia (Figure 4).

La seconde étape correspond en lectures préalables, approfondies et répétées des œuvres par l'adulte, qui permettent d'identifier en amont toute sa puissance, ses potentialités en terme d'apprentissages, notamment en géographie, et de prévoir les différentes lectures que pourront en faire les enfants. Ce travail de lecture fine porte à la fois sur le récit, le texte et les illustrations ; le maximum d'indices concernant les émotions est relevé.

Dans les œuvres du corpus retenu apparaissent des émotions géographiques fort variées. Certaines sont attendues : la grande tristesse face au départ forcé, l'excitation du voyage, un certain malaise, mal être dans des lieux nouveaux (la timidité notamment). D'autres le sont un peu moins : la nostalgie et la fierté à l'égard de son milieu d'origine, la colère voire la révolte face à l'indifférence, la recherche de la sécurité et de la tranquillité. L'une d'entre elles est assez originale et mérite d'être soulignée et discutée avec les enfants de manière approfondie : la détermination face aux difficultés et la volonté d'apprentissage qui passe par la (re)création d'un nouvel espace. 
Figure 4 : L'expérience de l'exil à partir d'un corpus d'œuvres de littérature de jeunesse

Sarah STEWART, David SMALL, La cabane d'Isabel, Paris, Syros, 2012, n. p.

Isabel quitte le Mexique avec sa famille pour aller s'installer aux Etats-Unis. Elle écrit

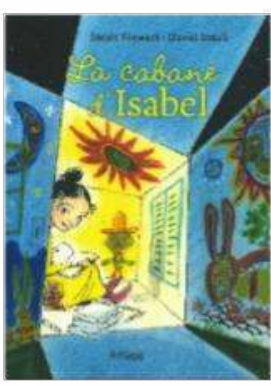
régulièrement à sa tante restée au pays et lui fait part de ses émotions. Très vite, elle veut apprendre sa nouvelle langue et surtout, se construire un espace à elle, une cabane en carton où elle pourra être tranquille et en sécurité. Elle trouvera le bonheur en invitant ses nouveaux amis dans sa cabane aux couleurs de son pays d'origine.
Sarah STEWART, David SMALL, Le jardin secret de Lydia, Paris, Syros, 2008, n. p.

Aux Etats-Unis en 1935, Lydia doit quitter sa famille et la campagne pour être accueillie en ville chez son oncle

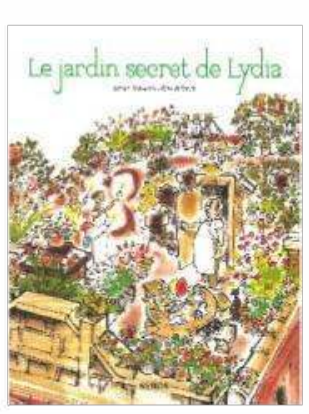
boulanger. Elle part jusqu'à ce que la situation de la famille s'arrange. Si elle aide son oncle à la boutique, elle entreprend en même temps de faire pousser des plantes avec des semences que lui envoie sa famille. Elle crée ainsi un extraordinaire jardin sur la terrasse d'un toit d'immeuble. En partageant ses plantes et ses connaissances Lydia répand sa bonne humeur.
Claudine GALEA, La fille qui parle à la mer / Le garçon au chien parlant, Arles, Rouergue, 2013, $31+30$ p.

Oyana et Loïc, deux enfants, se rencontrent sur la plage. Oyana s'y est échouée après une tempête subie sur un bateau où elle se trouvait avec sa famille et une

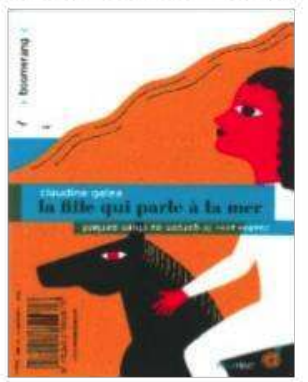
partie de son village. Tous avaient fui quelque temps plutôt leur pays en guerre. Mais en attendant le départ, Oyana, fille des montagnes, avait apprivoisé la mer et savait un peu nager les autres ont probablement péri en mer. Loïc, qui a l'habitude avec son chien de parcourir la plage à la recherche de trésors, y découvre ce matin-là Oyana et l'accueille dans sa famille; ils deviennent amis, peut-être davantage.
Gisèle PINEAU, Les Colères du volcan, Paris, Dapper, 2008 (2004), 140 p.

Cynthia habite en Guadeloupe ; elle se rend sur l'île voisine de Montserrat dans le cadre d'un

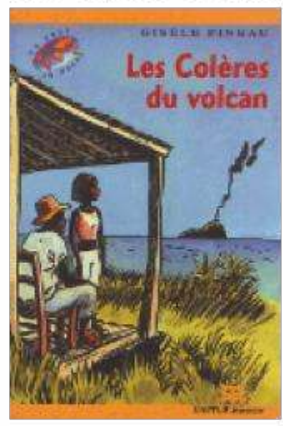
échange scolaire. Elle y découvre une île à la fois assez pauvre et très belle ; elle $s^{\prime} y$ fait une amie. Quelques semaines plus tard, le volcan de la petite île entre en éruption. Les habitants, dont l'amie de Cynthia, sont en grand danger et certains sont finalement forcés de s'exiler. Cynthia, sans nouvelles, s'inquiète beaucoup et se révolte face à l'indifférence générale.

\section{Nadine BRUN-COSME, Une si petite valise, Paris, L'école des loisirs, 2009, 64 p.}

D'abord de petits bonheurs en famille et puis soudain il faut partir, abandonner les habitudes. Les parents disent aux enfants qu'on ne peut prendre qu'une petite valise. Sur le bateau qui les emmène, les enfants ont des rapports difficiles les uns avec les autres et avec les adultes - ce qui les amène tour à tour à se cacher dans un coin sombre. Ils décident finalement de se montrer ce qu'ils ont choisi d'emporter et ils échangent des souvenirs et parlent de là-bas, de l'autre côté.

Réalisation : F. Cavaillé, 2016.

De manière générale, il apparaît assez clairement qu'une même structure-type des émotions est présente dans les différentes œuvres du corpus selon laquelle les personnages sont menés du malheur ou de l'inquiétude vers un bonheur ou un apaisement. Les personnages et les lecteurs - passent d'émotions de valence négative à des émotions de valence positive. Alors que l'expérience de l'exil est a priori négative, la valence positive des émotions l'emporte finalement dans chacun des récits. L'issue est réellement heureuse ou positivement ouverte. 
On retrouve là une structure-type des récits pour la jeunesse qui est rassurante, constructive. Les enfants ont surmonté les épreuves, ils ont réalisé des apprentissages fondamentaux, ils en sortent grandi. Ils s'en sortent mieux que les adultes ne pouvaient l'imaginer, ils s'en sortent parfois même mieux que les adultes eux-mêmes (Ziethen, 2012 : 88-89). L'exil d'Isabel apparait ainsi comme un modèle de réussite ${ }^{3}$. La lecture du corpus révèle par ailleurs, dans quatre documents sur cinq, que si les personnages enfants vivent des expériences positives, c'est grâce à la construction d'un espace original, ou bien à l'apprivoisement d'un milieu : une cabane, un jardin, la mer, un bateau. Dans l'album choisi, l'espace intime que se crée Isabel joue un rôle fondamental (il devient finalement le sujet principal de l'album), il est fabriqué à l'image du lieu d'origine perdu et devient le lieu d'accueil de ses nouveaux amis, autrement dit de son intégration réussie. La cabane d'Isabel et le jardin de Lydia deviennent des tiers-espaces réels faisant de l'exil une expérience qui non seulement peut être surmontée, mais peut même être enrichissante. Ces tiers-espaces originaux sont en effet créés dans l'espace d'accueil, comme de petites enclaves originales et personnalisées, de manière à garder un lien avec le pays d'origine (ils en renferment alors le souvenir et permettent de prolonger des habitudes anciennes) tout en étant des lieux de reconnaissance et d'intégration dans la nouvelle communauté.

De ce travail de lectures ressort par conséquent, un double questionnement concernant les émotions géographiques: il faudrait inévitablement essayer de comprendre et expliquer avec les enfants d'une part, la prégnance des émotions positives face à des expériences spatiales a priori difficiles, douloureuses et d'autre part le rôle éminemment constructif de certains lieux intimes ou privés dans ces mêmes situations difficiles.

La troisième étape, fondamentale, peut être concomitante au travail de lectures. Elle consiste en l'utilisation d'une matrice de lecture rassemblant des connaissances extérieures qui pourront directement nourrir le travail envisagé avec les œuvres. Les premières informations qui apparaissent nécessaires (et que recherchent fréquemment les enseignants - par exemple Go, 2009 : 24) ont trait aux auteurs et aux contextes de création de leurs œuvres. En sus de celles-ci, il est proposé d'éclairer les œuvres à partir de savoirs et d'approches scientifiques, issus de la géographie académique (ou qui ont trait à des objets, des problématiques de la géographie académique). L'objectif de cette " matrice de lecture géographique " est de rendre disponibles des acquis scientifiques permettant une lecture des œuvres littéraires informée par la géographie. C'est manifestement ce à quoi aspirait M. Brosseau lorsqu'il voulait "mettre en dialogue " roman et connaissance géographique : " [contraindre] un peu le texte à nous parler d'espace » et " [...] améliorer la compréhension du texte par un savoir géographique » (Brosseau, $1996: 62$ ).

\footnotetext{
${ }^{3}$ Cela est certainement compréhensible du point de vue des auteurs qui expliquent qu'ils se sont inspirés de I'histoire d'une personne réelle (Danielson, 2012 ; Lodge, 2012) mais cette vision est inacceptable selon d'autres : "In fact, in Isabel's world, there are no issues - no violence, no discrimination, no racism, no bullying, no taunting, and no suffering - because author, illustrator, and publisher do not have to see any. [...] Here, the author, illustrator and publisher have transformed Mexican and Mexican-American children's real lives, experiences and problems into a feel-good, pain-free picture book about assimilation for young readers who have never been forced to face the enormous difficulties associated with immigration or migration " (Slapin, 2015). ["En fait, dans le monde d'Isabel, il n'y a aucun problème - aucune violence, aucune discrimination, aucun racisme, aucune intimidation, aucune raillerie, et aucune souffrance - parce que l'auteur, l'illustrateur et l'éditeur n'en ont vu aucun. [...] Ici, l'auteur, l'illustrateur et l'éditeur ont transformé les vies réelles, les expériences et les problèmes des enfants mexicains et mexico-américains en un agréable et heureux album sur l'assimilation pour de jeunes lecteurs qui n'ont jamais été forcés d'affronter les grandes difficultés liées à l'immigration ou à la migration » - Traduction de l'auteur].
} 
Un exemple de matrice de lecture géographique est rapidement esquissé concernant la migration, l'exil (Figure 5) utilisant divers travaux (dont De Gourcy, 2013 : 52 ; Ma Mung, 2009). On peut voir concrètement comment cette matrice amène à dépasser une vision paradoxale et simplifiée du vécu exilaire. Elle présente l'ensemble du parcours migratoire laissant entendre qu'il peut comporter des étapes effectivement plus brutales, dangereuses, subies, etc. pour les migrants que celles que vivent les personnages des œuvres de jeunesse. Elle laisse toutefois également entendre que ce parcours peut s'inscrire dans une histoire collective, qu'il peut s'appuyer et développer l'autonomie individuelle, etc. Parmi les autres acquis scientifiques repris dans cette matrice, apparaît la (re)création d'espaces domestiques ou communautaires à l'image des lieux perdus comme possibilité du maintien de soi, du maintien de liens avec le territoire d'origine. Autrement dit, la conception d'une telle matrice fournit à l'adulte des repères structurés pour explorer, compléter, interpréter, prolonger les œuvres.

\section{Figure 5 : La préparation d'une matrice de lecture géographique de l'expérience de l'exil}

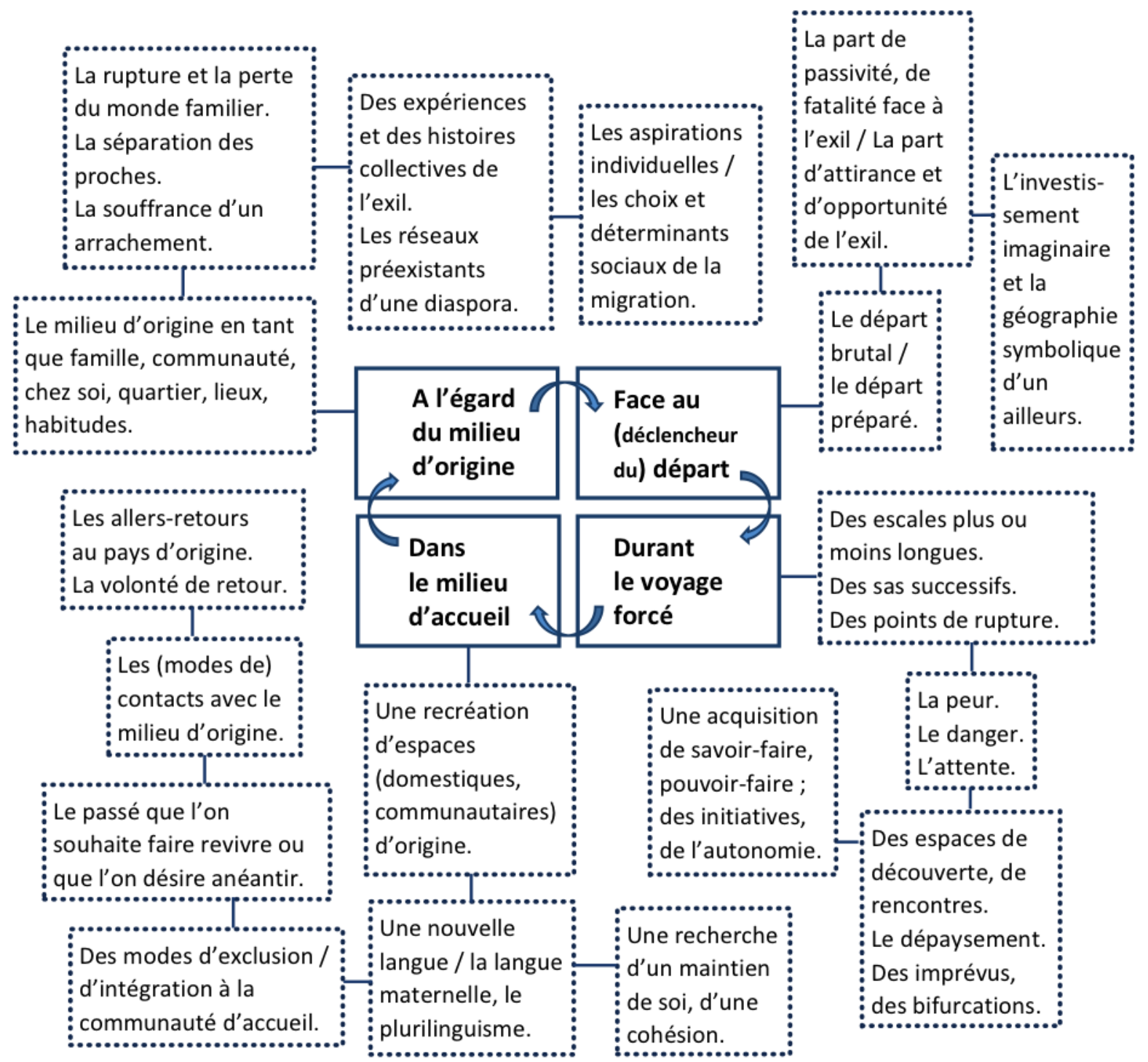

Réalisation : F. Cavaillé, 2016.

Enfin, la quatrième étape consiste en la conception des activités proposées aux enfants, qui 
sont de trois types (Figure 3) et qui recoupent les différentes émotions littéraires (Figure 2).

II s'agit de commencer par les activités de lecture en se concentrant sur les œuvres ellesmêmes et en essayant dans un premier temps de s'en tenir à une compréhension et une interprétation à partir des œuvres seules. L'objectif est une lecture d'immersion, empathique, voire fusionnelle pour plonger chaque enfant dans les expériences spatiales des personnages. Pour maîtriser le dévoilement des émotions probablement est-il préférable que ce soit l'adulte qui assure la lecture des œuvres. Une lecture oralisée voire théâtralisée peut particulièrement mettre en évidence les émotions présentes dans l'œuvre (comme on l'a laissé entendre plus haut, la mobilisation des différentes sensations s'avère pertinente : la voix lors de la lecture, la manipulation du livre, l'observation des illustrations, etc.). La mise en évidence des émotions littéraires (présentes dans l'œuvre et vécues lors de la lecture, par les lecteurs) doit aider l'enfant à identifier les émotions liées à l'exil et par là à comprendre l'expérience et les différentes étapes de la migration.

Dans le corpus présenté, au fil de chacun de ces récits, de chacune de ces expériences de l'exil, transparait une gamme nuancée d'émotions à la fois dans les situations du récit, au travers des vécus des personnages, dans la langue, par le vocabulaire, le style, la ponctuation, ou encore par le contenu et la nature des illustrations. Dans l'album choisi, les émotions sont ainsi très présentes dans la correspondance que la jeune héroïne entretient avec sa tante aimée, par exemple :

" 14 avril 1957 - Chère tante Lupita,

Nous ne sommes installés dans notre nouvelle maison que depuis dix jours mais nous avons défait presque tous les cartons. Je suis contente d'être à nouveau entourée de nos meubles et de nos affaires. Cette nuit, il est tombé beaucoup de neige. Quand je me suis réveillée, tout était blanc! [...]

L'anglais me vient encore difficilement, mais Chavo dit que ce sera de plus en plus facile avec le temps. Ces traces que tu vois sur le papier, ce sont mes larmes qui ont coulé. Une grosse tempête a soufflé sur le lac Michigan hier. J'avais laissé mon carton dehors et la pluie l'a détruit ».

Les émotions apparaissent également dans les illustrations au travers des postures des corps et surtout des visages. Après la grande tristesse de tous les personnages (que l'on imagine en pleurs) liée au départ, apparaissent tour à tour sur le visage d'Isabel la surprise de la découverte du nouveau pays, la timidité auprès des nouvelles connaissances, l'apaisement et finalement le bonheur dans une intimité accueillante (les émotions de l'héroïne peuvent être comparées avec celles des adultes et les personnages des autres œuvres du réseau). La palette des couleurs des lieux est en outre très différenciée : couleurs sombres du jour du départ, paysage gris du quartier ouvrier de l'arrivée s'opposant au paysage foisonnant et gai de la région d'origine, pastel du bien-être et éclats de couleurs vives du bonheur. Les expériences esthétiques sont alors en quelque sorte au service des expériences géographiques (Figure 6). 
Figure 6 : Les émotions géographiques liées à l'exil dans une œuvre de littérature de jeunesse

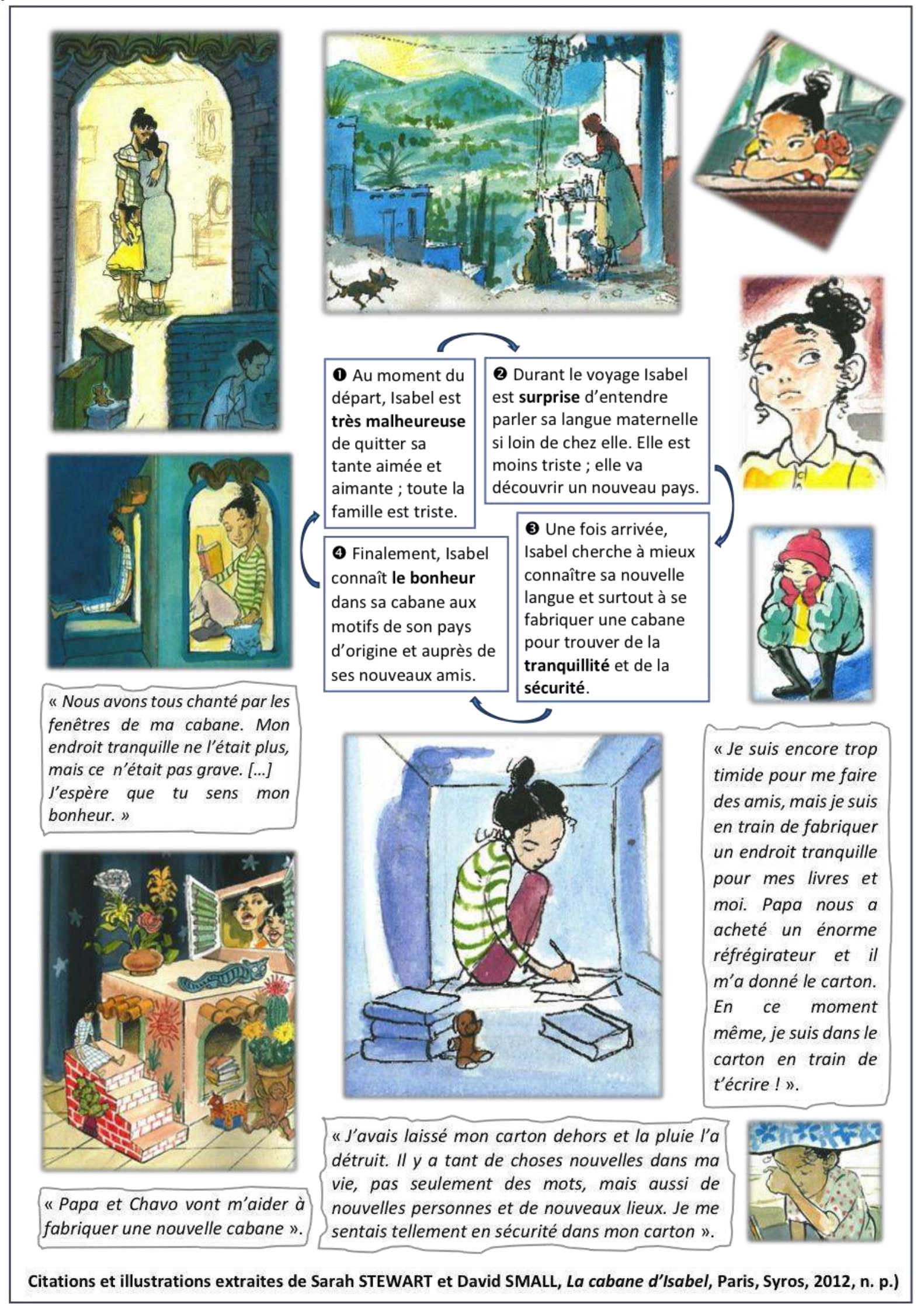

Réalisation : F. Cavaillé, 2016. 
Puis il convient à la fois de synthétiser, décomposer et réorganiser la lecture pour s'assurer que chaque enfant a compris les principaux moments du récit et du vécu de l'exil. Des activités pratiquées habituellement telles que, pour l'essentiel, des échanges oraux, en particulier les reformulations mais aussi des traces écrites succinctes, notamment dans un carnet de lecteur, peuvent se focaliser spécifiquement sur les émotions. Pour ce faire, il est envisagé ici de reconstituer un " scénario discursif des émotions " (Boquet, 2013, à partir de B. H. Rosenwein), c'est-à-dire de relever l'évolution des ressentis exprimés au fil du texte et des illustrations (Figure 6).

II est proposé en parallèle d'effectuer " une modélisation cartographique [...] [rendant] compte des espaces créés mais aussi de la conduite spatiale de celui qui parcourt ces espaces » (à partir de C. Meunier $2014: 181$ et suiv.). Il s'agit de bien identifier les espaces et spatialités présentes dans le récit (en étant attentif aux échelles et emboîtements d'espaces) et de les mettre explicitement en lien avec les émotions ressenties (Figure 7). Une telle synthèse - et sa représentation graphique - peut être élaborée avec les enfants ; elle peut être particulièrement pertinente pour comparer les expériences géographiques de plusieurs personnages d'œuvres mises en réseau (Tauveron, 2002a : 192-197).

Dans cette étape, par conséquent, c'est grâce à une lecture littéraire, grâce à des activités attentives à la langue, aux illustrations, etc., qui mettent explicitement en évidence les émotions, que les enfants - y compris d'ailleurs ceux qui peuvent être en difficulté ou réfractaires aux activités de lecture - pourront effectivement entrer dans cette expérience géographique sensible qu'est l'exil.

Dans un second temps, sont proposées aux enfants des activités visant la compréhension et l'interprétation à partir d'une lecture et une analyse maintenant nettement distanciées de l'œuvre. Dans toute cette phase, la matrice de lecture peut être directement utilisée. II faut d'abord pour l'essentiel faire appel aux questionnements et aux hypothèses d'interprétation des enfants. L'activité retenue peut être un débat d'interprétation structuré dans lequel les enfants sont plus particulièrement amenés à se questionner, à questionner l'adulte et à être questionnés par l'adulte pour élaborer des hypothèses explicatives. Face à l'expérience d'Isabel et de sa famille, il faut essayer de comprendre et d'expliquer pourquoi ils s'exilent, dans quelles conditions ils partent, ce qui cause la grande tristesse de toute la famille et d'Isabel en particulier, pourquoi Isabel souhaite autant se construire une cabane, comment elle la fabrique et pourquoi elle la fabrique ainsi, etc. Sont organisés des allers et retours fréquents entre moments de singularisation (où s'expriment les hypothèses et expériences personnelles des enfants) et moments de collectivisation (échanges dans lesquels se structurent des communautés émotionnelles ponctuelles). On peut noter au passage que ces échanges comportent une dimension et une fonction critiques qui amènent les enfants à présenter l'expérience de l'exil dans ses valences négatives - et peut-être de manière plus puissante que n'aurait pu l'expliciter l'œuvre elle-même.

Dans cette phase, les enfants vont d'eux-mêmes utiliser leurs propres vécus, leurs représentations et connaissances de l'éloignement, de la rupture, de leur besoin de lieux de tranquillité et de sécurité, de l'ambiguïté de l'isolement, etc. Ce sont alors les soubassements de leurs propres émotions, dans un mouvement réflexif, qu'ils vont mobiliser, partager et qu'ils vont de ce fait mettre au jour, pouvoir expliciter et formuler. Les enfants vont également raisonner, argumenter et problématiser pour élucider en tant que telle l'expérience du personnage. Par ce mouvement de la pensée, qui ne relève pas 
nécessairement et pas seulement d'une interrogation de soi, et qui peut notamment utiliser d'autres références littéraires, c'est une réflexion conceptuelle qui est élaborée (Chirouter, 2013 : 105 et suiv.). Dans ce double élan, ce que les enfants vont puiser en eux-mêmes et ce qu'ils vont faire émerger de leurs raisonnements, ce sont finalement les affects et les ressentis les plus profonds, les plus fondamentaux, qui sont au cœur des liens, intimes et sociaux, avec les lieux. Autrement dit, ce sont les sentiments géographiques, et non plus seulement les émotions, qui vont être mis au jour (Figure 7) : sentiment d'abandon, de bienêtre, d'appartenance et de reconnaissance par et dans les lieux. Finalement, les émotions sont des révélateurs des sentiments qui sont des révélateurs de la géographicité.

Figure 7 : Relevé du scénario des sentiments géographiques et des spatialités de La cabane d'Isabel.

\begin{tabular}{|c|c|c|c|c|c|}
\hline 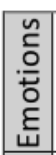 & $\begin{array}{l}\text { Malheur, tristesse } \\
\text { du départ. }\end{array}$ & $\begin{array}{l}\text { Surprise en } \\
\text { découvrant le } \\
\text { nouveau pays. }\end{array}$ & $\begin{array}{l}\text { Emotions } \\
\text { troublées. }\end{array}$ & $\begin{array}{l}\text { Tranquillité et } \\
\text { sécurité dans un } \\
\text { espace intime. }\end{array}$ & $\begin{array}{l}\text { Joie, bonheur avec } \\
\text { de nouveaux amis. }\end{array}$ \\
\hline$\tilde{y}$ & : & & & 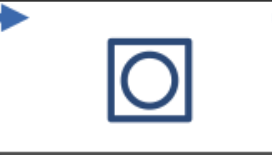 & \\
\hline . & $\begin{array}{l}\text { Sentiment } \\
\text { d'arrachement, } \\
\text { d'abandon. }\end{array}$ & $\begin{array}{l}\text { Sentiment } \\
\text { d'espoir? }\end{array}$ & $\begin{array}{l}\text { Réappropriation } \\
\text { de soi et des } \\
\text { lieux. }\end{array}$ & $\begin{array}{l}\text { Sentiment de } \\
\text { bien-être et de } \\
\text { sérénité. }\end{array}$ & $\begin{array}{l}\text { Sentiment } \\
\text { d'appartenance, } \\
\text { de reconnaissance. }\end{array}$ \\
\hline
\end{tabular}

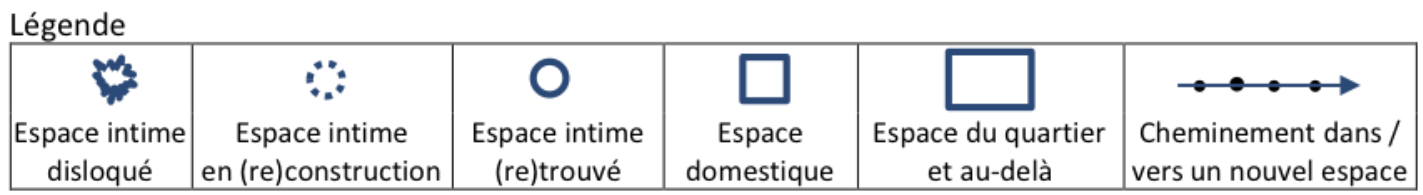

Réalisation : F. Cavaillé, 2016.

Peuvent ensuite être proposées d'autres activités nécessitant davantage de réflexion et d'autonomie de la part des enfants. Là encore, des pratiques éprouvées dans les classes semblent être particulièrement adaptées au travail sur les émotions - et le travail sur les émotions géographiques peut stimuler ces activités. II s'agit de proposer des écritures d'invention (ou des illustrations par le dessin) des "blancs " ou " trous " du récit (implicites, sauts dans le temps, dans l'espace, etc.), ou des suites de l'histoire (Tauveron, 2002b : 36). Par exemple il pourrait être demandé aux enfants d'imaginer des étapes du voyage d'Isabel ou ce qu'elle découvre dans son nouveau quartier de résidence ou bien encore de concevoir le retour d'Isabel dans son pays d'origine quelques années plus tard. Les échanges épistolaires - qui sont présents dans plusieurs œuvres du corpus - peuvent être investis par les enfants : il leur serait demandé de rédiger une lettre en adoptant le point de vue d'un autre acteur, notamment la tante ou un ami d'Isabel resté au pays. Un travail interdisciplinaire en français et en géographie se justifie pleinement ici, en particulier pour mobiliser dans la rédaction un lexique différencié des émotions. Il est probable que, projetés dans des cadres spatiaux relativement concrets (reconstituer un voyage, découvrir un quartier) les enfants sont d'autant plus à même d'effectuer des activités d'invention et d'écriture (Considère, Leduc, 2012).

Enfin, dans un troisième temps, peuvent être envisagées des activités de prolongement qui 
s'affranchissent encore davantage des œuvres pour présenter des apprentissages complémentaires, relevant plus directement ou plus classiquement de la géographie. II peut alors être effectué, en fonction des pistes d'interprétations des œuvres mises au jour un apport de connaissances spécifiques, le cas échéant en rapport avec les programmes d'enseignement, une recherche documentaire, de nature historique ou en lien éventuellement avec l'actualité, ou encore une enquête auprès d'acteurs (la reconstitution de parcours de migrants auprès des membres d'une association). Parmi les initiations visées spécifiquement en classe de géographie, on peut bien sûr envisager d'élaborer une cartographie des lieux présents dans les œuvres.

\section{Conclusion}

On le voit, (ap)prendre la géographie par les émotions véhiculées par la littérature, c'est-àdire par la fiction, par le récit d'invention, c'est accéder à une certaine densité de références, d'évènements, une mise en situation relativement complète et complexe, avec des contingences d' " univers possibles " faisant directement écho à notre monde ; autrement dit à de réelles expériences de vie, à partir d'immersions construites. C'est d'ailleurs l'apport de la fiction en tant que telle qu'il est intéressant de cerner (Cavaillé, 2016). La littérature rend de fait accessible aux enfants et disponible pour les adultes le champ d'une géographie sensible et met au jour une géographicité qui sans elle resteraient marginalisés et probablement ignorés. Les géographes peuvent conforter ce projet interdisciplinaire en enrichissant le travail sur les œuvres littéraires par des propositions précises, notamment en concevant des corpus d'œuvres et des matrices de lecture géographique comme cela a été envisagé ici.

L'apport de l'œuvre littéraire, comme celui des œuvres d'art, tient aux personnages singuliers, aux événements et aux situations particuliers (Jouve, 2010/2014 : 111-112, 155157) mais ces singularités recouvrent des expériences communes, récurrentes. Le lecteur a ainsi affaire à un cas singulier mais typique, en quelque sorte à un cas idéal-typique, paradigmatique (Rosat, 2006: 319-320). La fiction peut alors proposer le bénéfice des émotions, l'empathie, l'investissement, tout en maintenant une certaine distance du fait qu'elle est invention, (re)construction. (Ap)prendre la géographie par les émotions ou plutôt par les sentiments signifie donc pour les enfants et les adultes s'engager - parfois de manière réflexive - dans une pensée autour d'un objet médiatisé neutre, neutralisé, qui permet d'échanger des interprétations et de co-construire des références. Ainsi, par rapport à d'autres modes et supports d'apprentissage, l'œuvre de fiction de jeunesse et tout particulièrement l'album car il accentue probablement les émotions et génère un investissement plus important chez certains enfants :

"[...] est en quelque sorte à bonne distance [...] entre la trop grande proximité de l'expérience personnelle, qui empêche le recul et la réflexion, et le trop grand éloignement du concept qui empêche l'implication et l'engagement dans la pensée. Et c'est cette "bonne distance " qui occasionne le désir de parole [...] »(Demougin, 2008 : 269).

(Ap)prendre la géographie par les sentiments amène ainsi à apprendre autrement, à découvrir, comprendre et construire des connaissances autrement.

Il apparaît finalement qu'au-delà de l'enjeu de l'affirmation d'un champ ou d'objets particuliers de la géographie, c'est bien celui d'une éducation à la sensibilité dont il s'agit. II 
est probablement décisif que davantage d'enfants et d'élèves soient en mesure de décrypter et d'exprimer et donc d'imaginer de larges palettes émotionnelles, dont celles qui permettent de peindre les relations aux lieux, à l'espace.

"Si cette éducation [l'éducation morale] ne traite pas de sujets tels que : de quoi avoir peur ; contre quoi se mettre en colère ; quoi, le cas échéant, mépriser; où faire passer la limite entre ce qui est bonté et ce qui est sentimentalité stupide -je ne sais pas ce que signifie "éducation morale ». [...] Mais plus globalement, comme l'a bien vu Aristote, ce qui compte, c'est ce qu'il vaudrait mieux désigner par éducation des émotions que par apprentissage des principes [moraux] » (B. Williams repris par Tappolet, $2000: 255)$.

\section{Bibliographie}

AUDIGIER F. (2005), " Rien ne sert de nier les émotions, mais... ", in L. Lafortune et al. (dir.), Les émotions à l'école, Sainte-Foix, Presses de I'Université du Québec, pp. 73-99.

BEDARD M. (1987), " Plaidoyer de l'imaginaire pour une géographie humaniste ", Cahiers de géographie du Québec, vol.31, $n^{\circ} 82$, pp. 23-38, en ligne: https://www.erudit.org/revue/cgq/1987/v31/n82/021842ar.pdf

BLANC N. (2006), "Émotion et compréhension de textes », in N. Blanc (sous la dir. de), Émotion et cognition. Quand l'émotion parle à la cognition, Paris, Éditions in Press, pp. 123190.

BOQUET D. (2013), "Le concept de communauté émotionnelle selon B. H. Rosenwein ", Bulletin du centre d'études médiévales d'Auxerre, Hors-série $n^{\circ} 5$, en ligne: https://cem.revues.org/12535, accès le 28/01/2016.

BOUVERESSE J. (2006), "La littérature, la connaissance et la philosophie morale ", in S. Laugier (sous la dir. de), Ethique, littérature, vie humaine, Paris, PUF, pp. 95-145.

BROSSEAU M. (1996), Des romans-géographes. Essai, Paris, L'Harmattan, 246 p.

BRUGUIERE C., TRIQUET É. (2012), « Des albums de fiction réaliste pour problématiser le monde vivant ", Repères, $\mathrm{n}^{\circ} 45$, pp. 181-200.

CARIOU D. (2012), "Littérature de jeunesse et enseignement de l'histoire au cycle 3 ", Repères, $n^{\circ} 45, \mathrm{pp} .163-180$.

CAVAILLE F. (1999), L'expérience de l'expropriation, Paris, ADEF, 220 p.

CAVAILLE F. (2016), "Que peut la fiction pour la géographie ? Le rôle de la littérature de jeunesse dans les apprentissages ", Annales de géographie, $n^{\circ}$ 709-710, p. 246-271.

CHABANNE J.-C. et al., (2004), « Entre social, affects et langages, l'œuvre comme médiation. Prendre la littérature au sérieux dès l'école primaire ", Le français aujourd'hui, n¹45, pp. 7684.

CHIROUTER E. (2013), " Penser le monde grâce à la littérature : analyse d'une pratique à visée philosophique à l'école primaire ", Revue des sciences de l'éducation, vol. $39, n^{\circ} 1$, pp. 91-117.

CONSIDERE S., LEDUC C. (2012), " Fiction littéraire et géographie au cycle 3 », Repères, n45, pp. 181-200.

DANIELSON J. (2012), "Sarah Stewart and David Small Find 'The Quiet Place' ", Kirkus Reviews, 6/09/12, en ligne: https://www.kirkusreviews.com/features/sarah-stewart-anddavid-small-find-quiet-place/, dernier accès le 20/02/2016.

DARSEL S. (2012/2014), "Imagination narrative, émotions et éthique », Fabula / Les colloques, L'émotion, puissance de la littérature, en ligne: http://www.fabula.org/colloques/document2318.php, accès le 24/08/2015. 
DE GOURCY C. (2013), "Partir, rester, habiter : le projet migratoire dans la littérature exilaire ", Revue européenne des migrations internationales, vol. 29, n² ${ }^{\circ}$ pp. 43-57, en ligne : https://remi.revues.org/6631

DEMOUGIN F. (2008), "L'album lu : entre conscience et expérience », Modernités, $n^{\circ} 28$, pp. 265-276.

FEILDEL B. (2011), « Emotions et participation ou comment la délibération autour des projets d'aménagement participe de la construction du rapport affectif à l'espace ", Journée d'études sur les effets de la participation. GIS Participation du public, décision, démocratie participative, Paris, 19 p., en ligne: https://halshs.archives-ouvertes.fr/halshs-00694637, accès en août 2015.

FREMONT A. (2010), "État des lieux. À propos de l'espace vécu », Communications, vol. 2, n87, pp. 161-169.

FREVERT U. (2014), "Emotions perdues et émotions trouvées à l'ère contemporaine ", in Ambroise-Rendu A.-C., Demartini A.-E., H. Eck, N. Edelman (sous la dir. de), Emotions contemporaines. $\mathrm{XIX}^{\mathrm{e}}-\mathrm{XXI}^{\mathrm{e}}$ siècles, Paris, Armand Colin, pp. 45-68.

FROUILLOU L., (2011), « Les albums pour enfants et les géographies de l'enfance. L'exemple des représentations de la maison ", Carnets de géographes, ${ }^{\circ} 3$, décembre 2011, 14 p., en ligne: http://www.carnetsdegeographes.org/PDF/Rech 0302 Frouillou.pdf, dernier accès le $20 / 02 / 2016$.

GO H.-L., (2009), « Des ingénieries didactiques de l'œuvre (deux exemples en maternelle et en REGAD cycle 3) ", Education \& Didactique, vol. 3, n², pp. 7-45.

JAUBERT M., LALAGÜE-DULAC S., LOUICHON B. (2013) « Les fictions historiques : un objet littéraire, éditorial et scolaire qui interroge les frontières ", Repères, $n^{\circ} 48, p p .7-16$.

JOUVE V. (2010/2014), Pourquoi étudier la littérature ?, Paris, A. Colin, 222 p.

LANGLADE G. (2014), "La lecture subjective est-elle soluble dans l'enseignement de la littérature ? », Etudes de Lettres, vol. 1, n²95, pp. 47-62.

LE GUERN A.-L., THEMINES J.-F. (2011), « Des enfants iconographes de l'espace public urbain : la méthode du parcours iconographique ", Carnets de géographes, $n^{\circ} 3,19$ p., en ligne: http://www.carnetsdegeographes.org/PDF/Terrain 0302 Leguern Themines.pdf, dernier accès le 20/02/2016.

LEICHTER-FLACK F. (2012/2014), « Une question de vie ou de mort ? Des usages éthiques de l'émotion dans la fiction ", Fabula / Les colloques, L'émotion, puissance de la littérature, en ligne : http://www.fabula.org/colloques/document2328.php, accès le 24/08/2015.

LEVY B. (1997), "Géographie humaniste, géographie culturelle et littérature. Position épistémologique et méthodologique ", Géographie et cultures, $n^{\circ} 21, p p .27-44$.

LIVET P. (2004), "Actualité philosophique des émotions ", in A. Channouf et G. Rouan (éds), Emotions et cognitions, Bruxelles, De Boeck Université, pp. 41-71.

LODGE S. (2012), "Q \& A with Sarah Stewart and David Small », Publishers Weekly, 16/08/2012, en ligne : http://www.publishersweekly.com/pw/by-topic/childrens/childrensauthors/article/53558-q-a-with-sarah-stewart-and-david-small.html, dernier accès le 20/02/2016.

MA MUNG E. (2009), "Le point de vue de l'autonomie dans l'étude des migrations Internationales ", in F. Dureau et M. A. Hily, Les mondes de la mobilité, exploration d'un paradigme, Rennes, Presses de l'Université de Rennes, pp. 25-38.

MACE M., avec BARONI R., RODRIGUEZ A. (2014), "La lecture, les formes et la vie. Entretien », Etudes de lettres, n²95, pp. 165-180.

MANDROU R. (1959), "Pour une histoire de la Sensibilité », Annales. Économies, Sociétés, 
Civilisations, $14^{\mathrm{e}}$ année, $\mathrm{n}^{\circ} 3$, pp. 581-588.

MATTHEY L. (2008), "Quand la forme témoigne: réflexions autour du statut du texte littéraire en géographie ", Cahiers de géographie du Québec, vol. 52, n¹47, pp. 401-417, en ligne : https://www.erudit.org/revue/cgq/2008/v52/n147/029868ar.pdf

MEUNIER C., Les territoires de l'album, carnet de recherche, en ligne: http://Ita.hypotheses.org/

MEUNIER C. (2014), Quand les albums parlent d'Espace. Espaces et spatialités dans les albums pour enfants, Thèse de doctorat, Lyon, Ecole normale supérieure de Lyon, 576 p., en ligne : https://tel.archives-ouvertes.fr/tel-01127003/document, dernier accès le 20/02/2016. NAGY P. (2013), "Faire l'histoire des émotions à l'heure des sciences des émotions ", Bulletin du centre d'études médiévales d'Auxerre, Hors-série $n^{\circ} 5$, en ligne: http://cem.revues.org/12539, accès le 28/05/2015.

NONNON E. (2008), "Travail des mots, travail de la culture et migration des émotions : les activités de français comme techniques sociales du sentiment ", in M. Brossard et J. Fijalkov (dir.), Vygotski et les recherches en éducation et en didactiques, Pessac, Presses universitaires de Bordeaux, pp. 91-121.

PETIT A. (2014), « Des passions aux sentiments : le tournant affectif de la première moitié du XVIIle siècle ", Acta fabula, vol. $15, \quad n^{\circ} 4$, en ligne: http://www.fabula.org/acta/document8647.php, accès le 23/08/2015.

PUOZZO CAPRON I., PICCARDO E., (2013), "Au commencement était l'émotion : Introduction ", Lidil - Revue de linguistique et de didactique des langues, $n^{\circ} 48$, pp. 5-16, en ligne : https://lidil.revues.org/3308

ROSAT J.-J. (2006), Education politique et art du roman. Réflexions sur 1984, in S. Laugier (sous la dir. de), Ethique, littérature, vie humaine, Paris, PUF, pp. 309-330.

SLAPIN B. (2015), "The quiet place ", De Colores: The Raza Experience in Books for Children, en ligne: http://decoloresreviews.blogspot.fr/2015/11/the-quiet-place.html, dernier accès le 20/02/2016.

SYSSAU A. (2006), " La cognition a-t-elle besoin de l'émotion ou n'est-elle qu'une affaire de raison ? ", in N. Blanc (sous la dir. de), Émotion et cognition. Quand l'émotion parle à la cognition, Paris, Éditions in Press, pp. 51-62.

TAPPOLET C. (2000), Emotions et valeurs, Paris, PUF, 296 p.

TAUVERON C. (1999), " Comprendre et interpréter le littéraire à l'école : du texte réticent au texte proliférant ", Repères, $n^{\circ} 19$, pp. 9-38.

TAUVERON C. (dir.) (2002a), Lire la littérature à l'école. Pourquoi et comment conduire cet apprentissage spécifique ? De la GS a CM, Paris Hatier, 352 p.

TAUVERON C. (2002b), "La lecture comme jeu, à l'école aussi », in Tauveron C. (dir.), La lecture et la culture littéraires au cycle des approfondissements, Les Actes de la DESCO, Actes de l'université d'automne du 28 au 31 octobre 2002, pp. 23-40, en ligne: http://eduscol.education.fr/cid46316/la-lecture-comme-jeu-1-a-l-ecole-aussi.html, dernier accès le 20/02/2016.

ZIETHEN A. (2012), "La littérature pour la jeunesse ou l'art de "danser dans les chaînes" : trois textes sur la diaspora haïtienne en Amérique du Nord", Francophonies d'Amérique, n³3, pp. 79-94. 